\title{
Self-assembled organic and fullerene monolayers characterisation by two-colour SFG spectroscopy: a pathway to meet doubly resonant SFG process
}

\author{
C. Humbert*, Y. Caudano, L. Dreesen, Y. Sartenaer, A.A. Mani, C. Silien, \\ J.-J. Lemaire, P.A. Thiry, A. Peremans \\ Laboratoire de Spectroscopie Moléculaire de Surface, Facultés Universitaires Notre-Dame de la Paix, \\ 61 Rue de Bruxelles, B-5000 Namur, Belgium
}

Available online 18 August 2004

\begin{abstract}
Two-colour sum-frequency generation (two-colour SFG) spectroscopy was used to probe both vibrational and electronic properties of 1-dodecanethiol/Ag(llll 11 , $\mathrm{Au}\left(\begin{array}{lll}1 & 1 & 1\end{array}\right)$, and $\operatorname{Pt}\left(\begin{array}{lll}1 & 1 & 1\end{array}\right)$, of 5-[p-(6-mercaptohexoxy)-phenyl]-10,15,20-triphenylpor$\mathrm{phin} / \mathrm{Pt}\left(\begin{array}{lll}1 & 1 & 1\end{array}\right)$, and of $\mathrm{C}_{60} / \mathrm{Ag}\left(\begin{array}{lll}1 & 1 & 1\end{array}\right)$. The role of the various physical parameters determining the sum-frequency generation (SFG) intensity equation is highlighted. The enhancement of the non-linear second order susceptibility in the aforementioned interfaces is explained in terms of metal interband transition, molecular electronic transition and of electron-phonon coupling, respectively.
\end{abstract}

(C) 2004 Elsevier B.V. All rights reserved.

PACS: 42.62.Fi; 42.65.An; 42.65.Ky; 78.66.Qn; 78.66.Tr

Keywords: Sum-frequency generation; Vibration; Dodecanethiol; Porphyrin; Fullerene

\section{Introduction}

Sum-frequency generation spectroscopy (SFG) [1] is particularly well adapted to probe interfaces [2] due to its intrinsic sensitivity to non-centrosymmetric media. SFG photons are generated by mixing two laser beams at the same point of an interface. One is tuneable in the infrared $\left(\omega_{\text {Ir }}\right)$ spectral range, the other is fixed at $532 \mathrm{~nm}$ visible $\left(\omega_{\mathrm{Vis}}\right)$ wavelength. The SFG photons are detected at $\omega_{\mathrm{SFG}}=\omega_{\mathrm{Ir}}+\omega_{\mathrm{Vis}} . \mathrm{SFG}$

\footnotetext{
* Corresponding author. Tel.: +32-81-724706; fax: +32-81-724718.

E-mail address: christophe.humbert@fundp.ac.be (C. Humbert).
}

provides the interface vibrational fingerprint, as illustrated by various works on organic monolayers adsorbed on metals and insulators [3,4]. Today, thanks to the emergence of new optical parametric oscillators (OPOs) tuneable in the visible frequency range [5], SFG is extended to the so-called two-colour sumfrequency generation spectroscopy. It allows probing both vibrational and electronic properties as recently demonstrated [6,7]. Due to its strict selection rules, two-colour SFG is well suited to compete with other photon spectroscopies. These latter ones are generally limited to multilayer characterisation. They cannot provide simultaneous surface vibrational and electronic description at the submonolayer level. Two-colour 
SFG is an ideal complementary tool which can be used in real environment.

In this paper, we illustrate the versatility of twocolour SFG through experimental results on various adsorbed monolayers (dodecanethiol (DDT)/metals, porphyrin (TPD)/Pt, $\mathrm{C}_{60} / \mathrm{Ag}$ ) showing or not coupling between their electronic and vibrational structures. We also present basic theoretical guidelines, which highlight the experimental results and lead to a true DRSFG process in the fullerene case.

\section{Experimental setup}

Two-colour SFG is performed with laser beams generated by two OPOs built around $\mathrm{LiNbO}_{3}$ $\left(\mathrm{AgGaS}_{2}\right)$ and $\mathrm{BBO}$ non-linear crystals, tuneable from 2.5 to $4.2 \mu \mathrm{m}(4.2-8.2 \mu \mathrm{m})$ and from 415 to $720 \mathrm{~nm}$, respectively [5]. Both OPOs are synchronously pumped by an all-solid-state pulsed Nd:YAG laser operating at $25 \mathrm{~Hz}$ and delivering around 100 pulses of 12 ps duration per $1 \mu$ s laser burst. The infrared beam power at $3300 \mathrm{~cm}^{-1}$ is $30 \mathrm{~mW}$ (single pulse energy $\sim 24 \mu \mathrm{J}$ ) and the visible beam power at $500 \mathrm{~nm}$ is $15 \mathrm{~mW}$ (single pulse energy $\sim 12 \mu \mathrm{J}$ ). All beams are ppolarised and the spectral bandwidths of the infrared and visible ones are 2 and $3 \mathrm{~cm}^{-1}$, respectively.

We used the counter-propagating configuration in order to have a large separation between the SFG and the visible reflected beams [6]. Both the incident and the SFG beams propagate in the same plane, including the $\mathrm{z}$ sample surface normal. However, given the momentum conservation, the angular separation strongly depends on the infrared and visible wave vectors. In order to keep the SFG beam pointing constantly into a predetermined direction, the sample is mounted on a computer-driven rotary stage. The SFG photons were collected by a photomultiplier tube after spatial and spectral filtering in a home-made monochromator. Each SFG spectrum is normalised with respect to the visible and infrared intensities (see Eq. (1)) to take into account the fluctuations of the laser beams.

\section{Sample preparation}

All the single crystal surfaces were polished to a mirror-like finish. The $\operatorname{Pt}\left(\begin{array}{lll}1 & 1 & 1\end{array}\right)$ surface was flame- annealed during $10 \mathrm{~min}$ and then protected by a droplet of ultrapure water (resistivity $>18 \mathrm{M} \Omega \mathrm{cm}$ ), before immersion in the appropriate organic solution. The $\mathrm{Au}\left(\begin{array}{lll}1 & 1 & 1\end{array}\right)$ surface was chemically etched in a (3:1) $\mathrm{H}_{2} \mathrm{SO}_{4}: \mathrm{H}_{2} \mathrm{O}_{2}$ solution while $\mathrm{Ag}\left(\begin{array}{lll}1 & 1 & 1\end{array}\right)$ was rinsed with ethanol. The DDT self-assembled monolayers (SAMs) were prepared by immersing the substrates into a $1 \mathrm{mM}$ absolute ethanolic solution of $\mathrm{SH}\left(\mathrm{CH}_{2}\right)_{11} \mathrm{CH}_{3}$ (Aldrich), during $18 \mathrm{~h}$. The porphyrin SAMs were prepared by immersing the $\operatorname{Pt}\left(\begin{array}{lll}1 & 1 & 1\end{array}\right)$ into a $1 \mathrm{mM}$ dichloromethane solution of TPD, for $12 \mathrm{~h}$ in the dark. $\mathrm{C}_{60}$ monolayers were obtained by sublimating in ultra-high vacuum by evaporating the fullerene from a Ta crucible, while keeping the $\operatorname{Ag}\left(\begin{array}{lll}1 & 1 & 1\end{array}\right)$ substrate at $550 \mathrm{~K}$ in order to prevent multilayer growth. All measurements were carried out at ambient air.

\section{Results and discussion}

In keeping with previous works, the SFG intensity [8] is defined by:

$I\left(\omega_{\mathrm{SFG}}\right)=\frac{\omega_{\mathrm{SFG}}^{2}}{c^{2}} A_{\text {Fresnel }}\left|\chi_{\mathrm{S}}^{(2)}\right|^{2} I\left(\omega_{\mathrm{Ir}}\right) I\left(\omega_{\mathrm{Vis}}\right)$,

assuming that a single component of the interface second order non-linear susceptibility $\chi_{\mathrm{S}}^{(2)}$ dominates the SFG signal. $I\left(\omega_{\text {Ir }}\right)$ and $I\left(\omega_{\text {Vis }}\right)$ are the intensities of the two incident laser beams and $c$ is the light velocity. After normalisation by these parameters, the SFG intensity depends only on $\omega_{\mathrm{SFG}}, A_{\text {Fresnel }}$ (Fresnel factors) and $\chi_{\mathrm{S}}^{(2)}$. We further normalised all spectra with respect to $\omega_{\mathrm{SFG}}^{2}$ because its variations are not negligible when the visible wavelength is changed. The measurements described hereafter intent to enlighten the role of $A_{\text {Fresnel }}$ and $\chi_{\mathrm{S}}^{(2)}$.

\subsection{Influence of the substrate electronic structure: DDT/Ag, Au, and Pt}

In the SFG spectra presented in Fig. 1, we made measurements at four different incident visible wavelengths $(450,500,550,650 \mathrm{~nm})$. The most drastic changes are observed for DDT/Au(1 11 1) (Fig. 1B). Indeed, the mean SFG intensity reaches a maximum at $\sim 550 \mathrm{~nm}$. The $\mathrm{CH}_{3}$ symmetric $\left(2875 \mathrm{~cm}^{-1}\right)$, Fermi resonance $\left(2936 \mathrm{~cm}^{-1}\right)$ and asymmetric $\left(2963 \mathrm{~cm}^{-1}\right)$ 

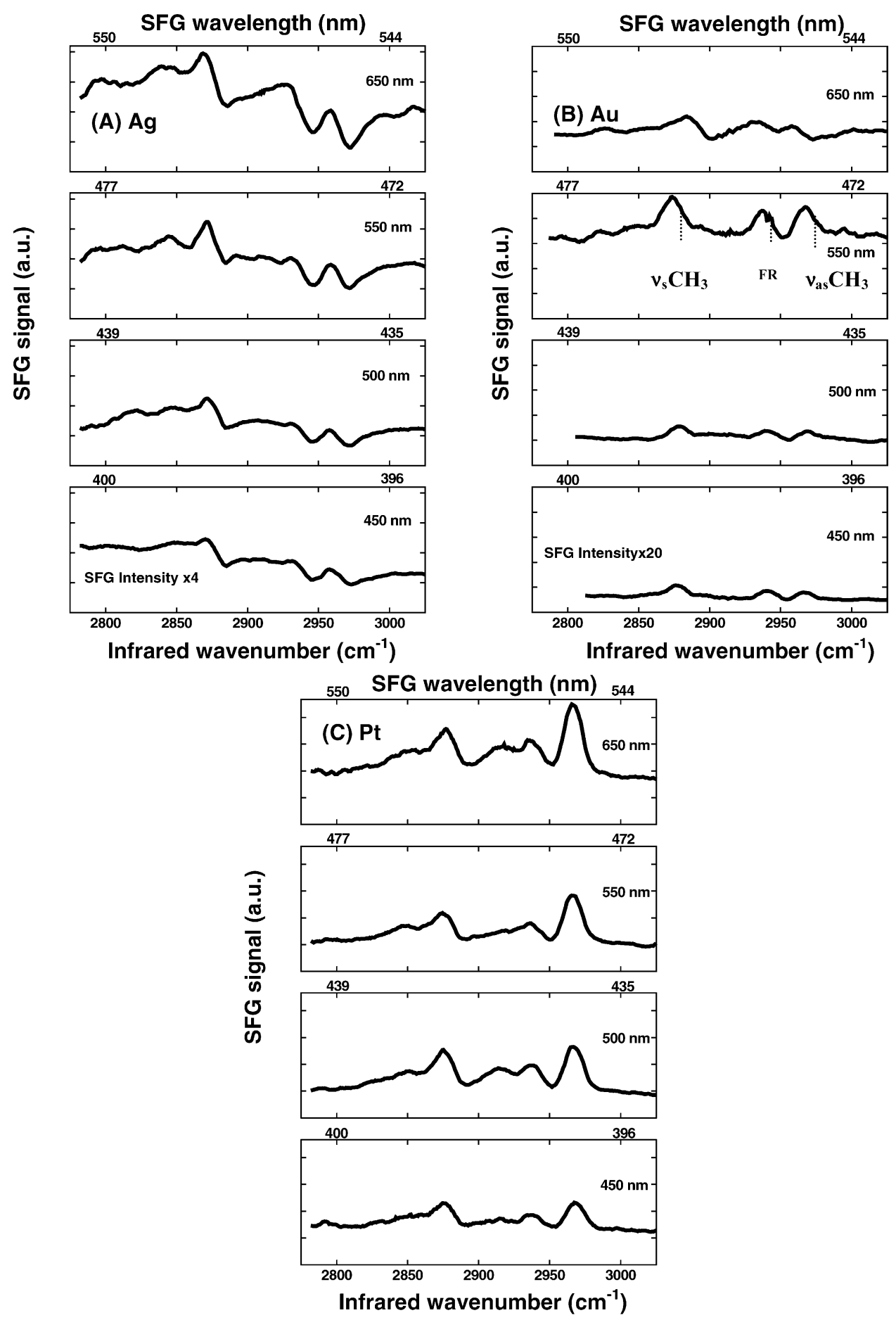

Fig. 1. SFG spectra of 1-dodecanethiol monolayer adsorbed on $\operatorname{Ag}\left(\begin{array}{lll}1 & 1 & 1\end{array}\right)$ (A), $\operatorname{Au}\left(\begin{array}{llll}1 & 1 & 1\end{array}\right)$ (B), and $\operatorname{Pt}\left(\begin{array}{lll}1 & 1 & 1\end{array}\right)$ (C). 


\section{$\left|X_{N R}^{(2)}\right|^{2}$ (a.u.)}
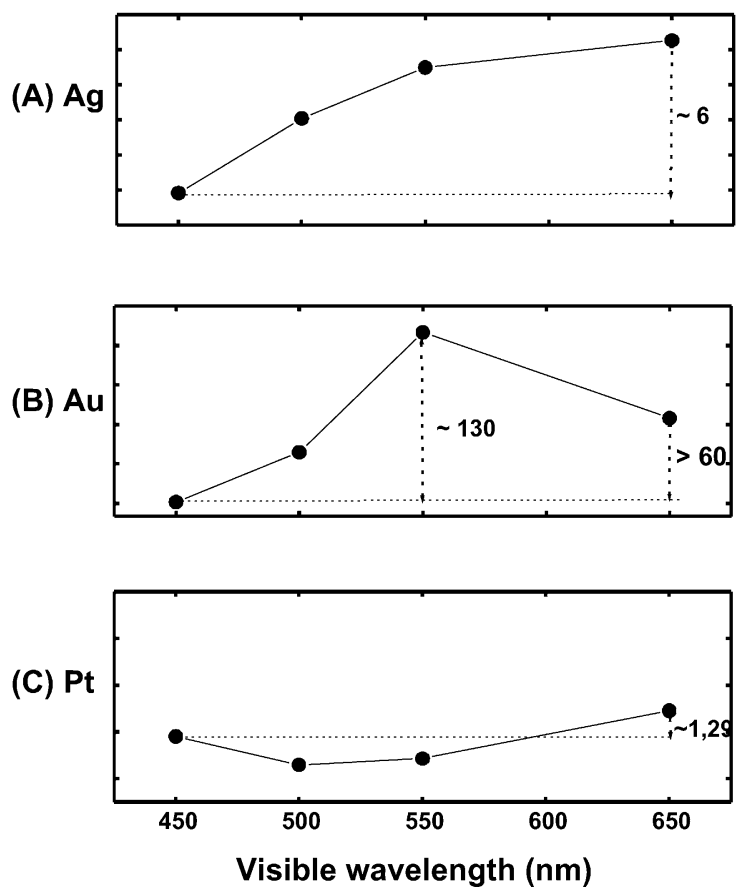

A Fresnel (a.u.)
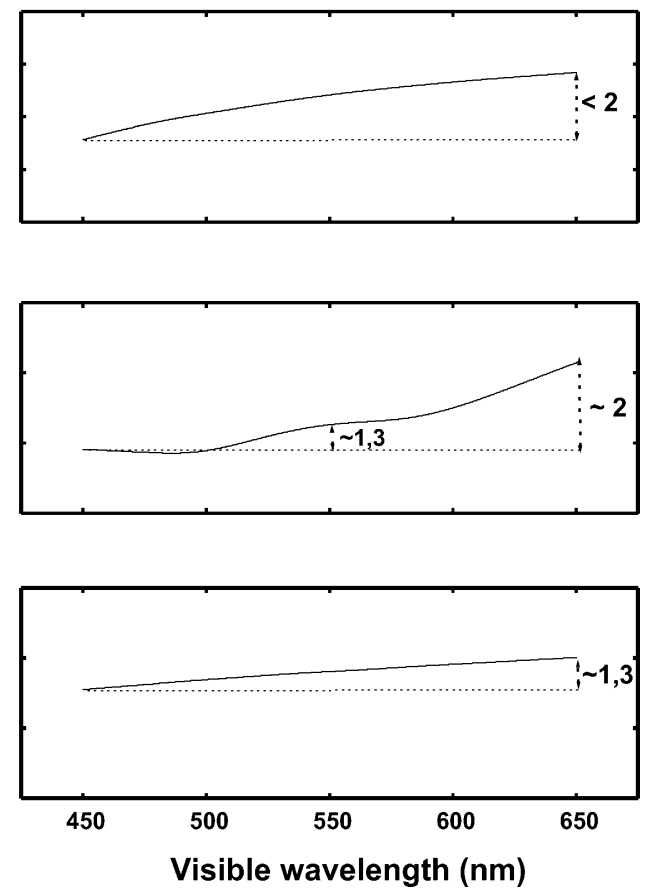

Fig. 2. Linear $\left(A_{\text {Fresnel }}\right)$ and non-linear $\left(\left|\chi_{\mathrm{NR}}^{(2)}\right|^{2}\right)$ SFG contributions of $\operatorname{Ag}\left(\begin{array}{lll}1 & 1 & 1\end{array}\right)$ (A), $\operatorname{Au}\left(\begin{array}{lll}1 & 1 & 1\end{array}\right)(\mathrm{B})$, and $\operatorname{Pt}\left(\begin{array}{lll}1 & 1 & 1\end{array}\right)$ (C).

stretching modes of the terminal group are peak-shaped for wavelengths in the blue $(450 \mathrm{~nm})$. They exhibit a spectral shape intermediate between valley and peak for wavelengths in the red

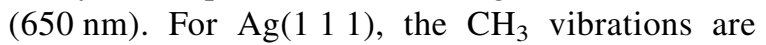
dip-shaped, while the mean SFG intensity slightly increases with the visible wavelength (Fig. 1A). For Pt(l 111$)$, all vibration modes are peak-shaped whatever the visible wavelength. In this case, the mean SFG intensity does not evolve significantly (Fig. 1C).

Neglecting the SAMs thin layers, we first evaluate $A_{\text {Fresnel }}$ for the air-metal interfaces. From Eq. (1), $A_{\text {Fresnel }}$ is a linear contribution to the SFG signal, which develops in terms of the interface Fresnel factors, for all the wavelengths involved in the SFG process:

$A_{\text {Fresnel }}=\frac{\left|F\left(\omega_{\mathrm{SFG}}\right)\right|^{2}\left|F\left(\omega_{\mathrm{Vis}}\right)\right|^{2}\left|F\left(\omega_{\mathrm{Ir}}\right)\right|^{2}}{4 \cos ^{2} \theta_{\mathrm{SFG}}}$.
$F\left(\omega_{\mathrm{SFG}}\right), F\left(\omega_{\mathrm{Vis}}\right), F\left(\omega_{\mathrm{Ir}}\right)$ are the second rank tensors and are diagonal for isotropic media [8]. They depend on the wave vectors and on the evolution of the dielectric properties in reflection, transmission, and at the interface separation. The calculated values of $A_{\text {Fresnel }}$ are displayed in Fig. 2A-C, for ppp polarisation, for $\operatorname{Ag}\left(\begin{array}{lll}1 & 1 & 1\end{array}\right), \operatorname{Au}\left(\begin{array}{lll}1 & 1 & 1\end{array}\right), \operatorname{Pt}\left(\begin{array}{lll}1 & 1 & 1\end{array}\right)$, respectively. The dielectric functions were taken from Ref. [9] in the investigated spectral range. As illustrated in Fig. 2B, $A_{\text {Fresnel }}$ does not explain the evolution of the SFG intensity on $\mathrm{Au}\left(\begin{array}{lll}1 & 1 & 1\end{array}\right)$. It should thus be related to $\chi_{\mathrm{S}}^{(2)}$. Eq. (1) can be rewritten as:

$$
\begin{aligned}
I\left(\omega_{\mathrm{SFG}}\right) & \propto\left|\chi_{\mathrm{S}}^{(2)}\right|^{2} \propto\left|\chi_{\mathrm{NR}}^{(2)}+\chi_{\mathrm{R}}^{(2)}\right|^{2} \\
& \propto|| \chi_{\mathrm{NR}}^{(2)}\left|\mathrm{e}^{\mathrm{i} \Phi}+\sum_{q=1}^{3} \frac{\left|a_{q}\right| \mathrm{e}^{\mathrm{i} \varphi_{q}}}{\omega_{q}-\omega_{\mathrm{Ir}}+\mathrm{i} \Gamma_{q}}\right|^{2} .
\end{aligned}
$$

$\chi_{\mathrm{NR}}^{(2)}$ and $\chi_{\mathrm{R}}^{(2)}$ are the second order non-linear susceptibilities of the substrate (infrared non-resonant) and of 
the adsorbate (infrared resonant). The non-resonant substrate contribution can be treated like a scalar. $\chi_{\mathrm{R}}^{(2)}$ is a third rank tensor whose contribution to the ppp SFG signal arise only from the $\chi_{z z z}, \chi_{z x x}, \chi_{z x z}$, and $\chi_{x x z}$ components on isotropic surfaces. However, due to the important values of the metal dielectric constants, the molecular adsorbate response is usually dominated by $\chi_{z z z}$ since the parallel components of the electric field are screened (Fresnel factor $F_{z z} \gg F_{x x}, F_{y y}$ ). The vibration modes $\left(\omega_{q}\right)$ of DDT are described by a sum on three Lorentzian oscillators with damping constants, $\Gamma_{q} \cdot a_{q}$ is the complex oscillator strength, which contains a Raman contribution due to the visible and SFG beams. For DDT molecules, only the infrared resonant terms must be considered, given that the Raman contribution of the visible and SFG beams, contained in $a_{q}$, does not depend on the visible wavelength.

The phase shift $\Phi-\varphi_{q}$ in Eq. (3) explains the interference pattern between the substrate and adsorbate non-linear responses that generates the different spectral shapes in Fig. 1A-C. We have simulated the metal non-linear contribution $\left|\chi_{\mathrm{NR}}^{(2)}\right|^{2}$ using Eq. (3) and the metal non-linear susceptibility parameters $\left(\left|\chi_{\mathrm{NR}}^{(2)}\right|\right.$, $\Phi)$, reported in a previous work [10]. $\left|\chi_{\mathrm{NR}}^{(2)}\right|^{2}$ is compared to $A_{\text {Fresnel }}$ in Fig. 2. This leads us to conclude that there is no significant contribution of $A_{\text {Fresnel }}$ to the SFG signals whatever the substrate. Nevertheless, for $\mathrm{Au}\left(\begin{array}{lll}1 & 1 & 1\end{array}\right)$ in Fig. 2B, the enhancement of the SFG response at $550 \mathrm{~nm}$ visible wavelength is explained by the evolution of $\left|\chi_{\mathrm{NR}}^{(2)}\right|^{2}$. Indeed, it is related to an interband $\mathrm{s}-\mathrm{d}$ electronic transition of Au that resonates when the corresponding SFG photon wavelength is $480 \mathrm{~nm}(2.58 \mathrm{eV})$ [10]. No similar enhancement is observed on $\operatorname{Ag}\left(\begin{array}{lll}1 & 1 & 1\end{array}\right)$ given that the SFG wavelength range required $(315 \mathrm{~nm}, 3.93 \mathrm{eV})$ is not accessible here (Fig. 2A). The slight growth of the mean intensity can be related to an increase of the Ag metal reflectance [11]. For $\operatorname{Pt}\left(\begin{array}{lll}1 & 1 & 1\end{array}\right)$, no distinction can be made between $A_{\text {Fresnel }}$ and $\left|\chi_{\mathrm{NR}}^{(2)}\right|^{2}$ (Fig. 2C). The non-linear activity of the substrate is so weak that the vibration modes are always peak-shaped.

\subsection{Influence of the adsorbate electronic structure: $T P D / P t$}

We now consider an organic adsorbate (TPD) showing electronic activity in the probed energy range. The
SFG spectra of TPD/Pt(llll 11 ) measured at four different visible wavelengths $(480,510,520,550 \mathrm{~nm})$ are shown in Fig. 3A. The pyrrole $\left(3060 \mathrm{~cm}^{-1}\right)$ and phenyl $\left(3030 \mathrm{~cm}^{-1}\right) \mathrm{CH}$ stretching modes, as well as the $\mathrm{CH}_{2}$ symmetric $\left(2865 \mathrm{~cm}^{-1}\right)$ and asymmetric $\left(2925 \mathrm{~cm}^{-1}\right)$ stretching modes are detected on the spectra. Contrary to the DDT/Pt(llll 111$)$ interface, we observe that the average SFG intensity evolves and reaches a maximum at $510 \mathrm{~nm}(\mathrm{SFG} \sim 435 \mathrm{~nm})$. It comes from the so-called Soret band $\left(\mathrm{S}_{0}-\mathrm{S}_{2}\right)$ of the TPD porphyrin moiety [12] whose wavelength matches the SFG one at $435 \mathrm{~nm}$. In this case, the second order non-linear susceptibility of the interface responsible for the SFG process is only the adsorbate one:

$\chi_{\mathrm{S}}^{(2)}=\chi_{\mathrm{R}}^{(2)}=\chi_{\mathrm{vib}}^{(2)}+\chi_{\mathrm{e}}^{(2)}$.

$\chi_{\mathrm{vib}}^{(2)}$ (infrared resonant Lorentzian term of the molecule) contains a similar contribution as for DDT molecules, modelling the existence of the $\mathrm{CH}_{2}$ and $\mathrm{CH}$ infrared vibration modes. $\chi_{\mathrm{e}}^{(2)}$ (SFG resonant Lorentzian term of the molecule) represents the electronic Soret band, which explains the stronger enhancement of the mean SFG intensity for TPD/ $\operatorname{Pt}\left(\begin{array}{lll}1 & 1 & 1\end{array}\right)[12,13]$. We also observe an evolution with the visible wavelength of the relative intensities of the $\mathrm{CH}_{2}$ and $\mathrm{CH}$ vibrations. It could be related to their different electronic activity due to their position, either on the alcane chain or on the phenyl and pyrrole groups where the Soret band transition originates, respectively. However, the combination of the two second order non-linear molecular susceptibilities in Eq. (4) does not exhibit a true DRSFG process: it could only occur if the infrared vibration mode was coupled to an electronic transition.

\subsection{Electron-phonon coupling or DRSFG process: $C_{60} / A g\left(\begin{array}{lll}1 & 1 & 1\end{array}\right)$}

$\mathrm{C}_{60}$ monolayers adsorbed on $\operatorname{Ag}\left(\begin{array}{lll}1 & 1 & 1\end{array}\right)$ were measured at four different visible wavelengths $(458,532$, $568,647 \mathrm{~nm}$ ). The spectra are shown in Fig. 3B. We observe a large enhancement of the SFG intensity of the $A_{\mathrm{g}}(2)$ pentagonal pinch vibration $\left(\sim 1460 \mathrm{~cm}^{-1}\right)$ at $532 \mathrm{~nm}$ visible wavelength. Although the $A_{\mathrm{g}}(2)$ mode is only Raman-active in isolated $\mathrm{C}_{60}$, it exhibits an important infrared activity as well when the fullerene 
(A) TPD/Pt(111)

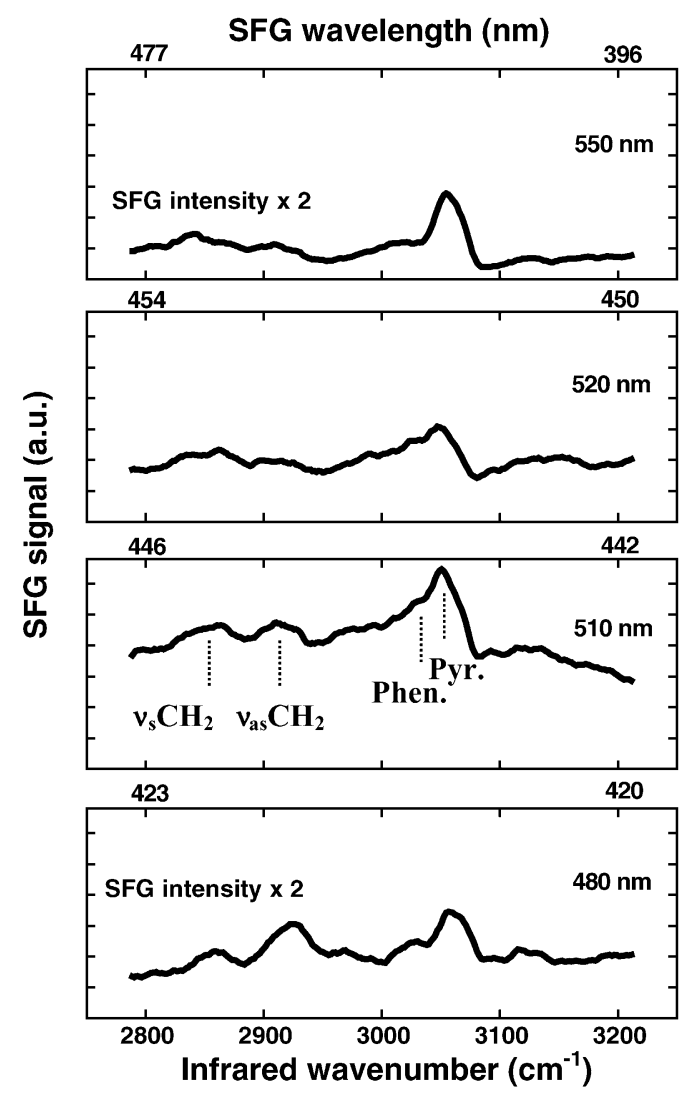

(B) $\mathrm{C}_{60} / \mathrm{Ag}(111)$

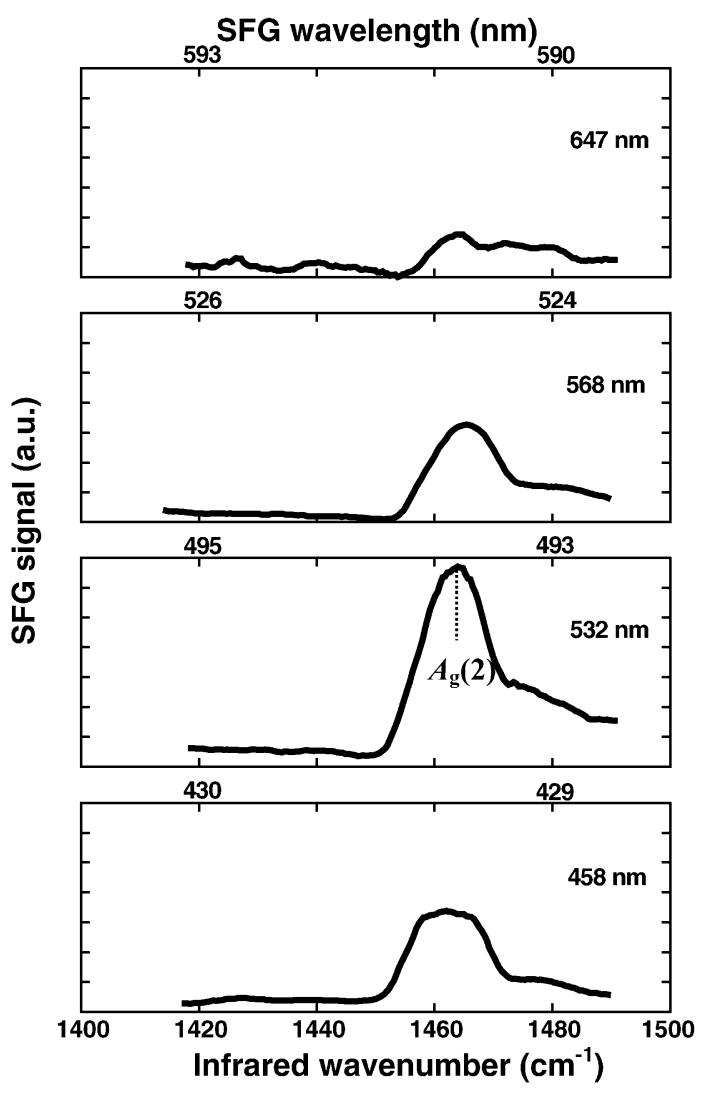

Fig. 3. SFG spectra of 5-[p-(6-mercaptohexoxy)-phenyl]-10,15,20-triphenylporphine/Pt(1 11 1) (A) and of $\mathrm{C}_{60} / \operatorname{Ag}\left(\begin{array}{lll}1 & 1 & 1\end{array}\right)$ (B).

is adsorbed on a metal surface [14,15]. It allows its detection by SFG. The dipolar activity arises from the coupling between the vibration and electronic states localised at the interface $[14,15]$. Here, the non-resonant contribution of $\operatorname{Ag}\left(\begin{array}{lll}1 & 1 & 1\end{array}\right), \chi_{\mathrm{NR}}^{(2)}$, and the electronic resonant contribution of the adsorbate, $\chi_{\mathrm{e}}^{(2)}$, are unable to explain the enhancement at $532 \mathrm{~nm}$ visible wavelength. For $\mathrm{C}_{60} / \operatorname{Ag}\left(\begin{array}{lll}1 & 1 & 1\end{array}\right), \chi_{\mathrm{S}}^{(2)}$ reads $[13,16]$ :

$$
\begin{aligned}
\chi_{\mathrm{S}}^{(2)}= & \chi_{\mathrm{R}}^{(2)}=\chi_{\mathrm{DRSFG}}^{(2)} \propto \frac{1}{\left(\omega_{\mathrm{Ir}}-\omega_{A_{\mathrm{g}}(2)}+\mathrm{i} \Gamma_{A_{\mathrm{g}}(2)}\right)} \\
& \times \frac{1}{\left(\omega_{\mathrm{SFG}}-\omega_{\mathrm{el}}+\mathrm{i} \Gamma_{\mathrm{el}}\right)} .
\end{aligned}
$$

A strong SFG intensity is only detected when the infrared $\left(\sim 1460 \mathrm{~cm}^{-1}\right)$ and SFG wavelengths ( $\sim 94 \mathrm{~nm})$ matches both the interface $A_{\mathrm{g}}(2)$ pentago- nal pinch mode and the specific HOMO (highest occupied MO)-LUMO (lowest unoccupied MO) electronic transition, respectively [16]. Indeed, at $494 \mathrm{~nm}$ SFG wavelength, the energy of the SFG photons is about $2.5 \mathrm{eV}$. This value is close to the $2.2 \pm 0.3 \mathrm{eV}$ energy gap between the HOMO and LUMO derived states measured for $\mathrm{C}_{60} / \mathrm{Ag}\left(\begin{array}{lll}1 & 0 & 0\end{array}\right)$ [17]. Henceforth, a true DRSFG process occurs at this interface.

\section{Conclusion}

Two-colour SFG was used to characterise both the electronic and vibrational properties of SAMs and fullerene monolayers adsorbed on different metallic substrates. We probed interfaces where interband metallic transitions [DDT/Au(l 11 l $)$ ], molecular 
transitions [porphyrin derivative/ $\left.\operatorname{Pt}\left(\begin{array}{lll}1 & 1 & 1\end{array}\right)\right]$ and electron-phonon couplings $\left[\mathrm{C}_{60} / \mathrm{Ag}\left(\begin{array}{lll}1 & 1 & 1\end{array}\right)\right]$ enhance the SFG signal for particular wavelengths: metal interband $\mathrm{s}-\mathrm{d}$, Soret band electronic and interband involving the LUMO electronic transitions, respectively. Basic theoretical developments showed that, among the systems presented here, only the fullerene case should be considered as a true DRSFG process.

\section{Acknowledgements}

$\mathrm{CH}$ and $\mathrm{CS}$ are Scientific Research Workers of the Belgian National Fund for Scientific Research (FNRS). YC and AP are Postdoctoral Researcher and Research Associate of the FNRS, respectively. This work is supported by the Ministry of the Walloon Region (Belgium) and the Interuniversity Research Program 5/1 on "Quantum size effects in nanostructured materials", initiated by the Belgian Office for Scientific, Technical and Cultural Affairs (OSTC).

\section{References}

[1] P. Guyot-Sionnest, J.H. Hunt, Y.R. Shen, Phys. Rev. Lett. 59 (1987) 1597.

[2] K.B. Eisenthal, Chem. Rev. 96 (1996) 1343.

[3] C.D. Bain, Faraday Trans. 91 (1995) 1281.
[4] T. Dellwig, G. Rupprechter, H. Unterhalt, H.-J. Freund, Phys. Rev. Lett. 85 (2000) 776.

[5] A.A. Mani, L. Dreesen, C. Humbert, P. Hollander, Y. Caudano, P.A. Thiry, A. Peremans, Surf. Sci. 502-503 (2002) 261.

[6] C. Humbert, L. Dreesen, A.A. Mani, Y. Caudano, J.-J. Lemaire, P.A. Thiry, A. Peremans, Surf. Sci. 502-503 (2002) 203.

[7] M.B. Raschke, Y.R. Shen, M. Hayashi, S.H. Lin, Chem. Phys. Lett. 359 (2002) 367.

[8] T.F. Heinz, Non-linear Surface Electromagnetic Phenomena, Elsevier, Amsterdam, 1991, Chapter 5.

[9] E.D. Palik, Handbook of Optical Constants of Solids, Academic Press, London, 1985.

[10] L. Dreesen, C. Humbert, M. Celebi, J.-J. Lemaire, A.A. Mani, P.A. Thiry, A. Peremans, Appl. Phys. B: Lasers Opt. 74 (2002) 621.

[11] H. Ehrenreich, H.R. Philipp, Phys. Rev. 128 (1962) 1622.

[12] C. Humbert, L. Dreesen, S. Nihonyanagi, T. Masuda, T. Kondo, A.A. Mani, K. Uosaki, P.A. Thiry, A. Peremans, Appl. Surf. Sci. 212-213 (2003) 797.

[13] S.H. Lin, M. Hayashi, R. Islampour, J. Yu, D.Y. Yang, G.Y.C. Wu, Physica B 222 (1996) 191.

[14] A. Peremans, Y. Caudano, P.A. Thiry, P. Dumas, W.-Q. Zheng, A. Le Rille, A. Tadjeddine, Phys. Rev. Lett. 78 (1997) 2999.

[15] P. Rudolf, R. Raval, P. Dumas, G.P. Williams, Appl. Phys. A 75 (2002) 147.

[16] Y. Caudano, C. Silien, C. Humbert, L. Dreesen, A.A. Mani, P.A. Thiry, A. Peremans, J. Electron Spectr. Relat. Phenomena 129 (2003) 139.

[17] M. Grobis, X. Lu, M.F. Crommie, Phys. Rev. B 66 (2002) 161408(R). 